\title{
ERGODIC SEMIGROUPS OF EPIMORPHISMS
}

\author{
BY
}

DANIEL BEREND

\begin{abstract}
The conditions for ergodicity of semigroups of epimorphisms of compact groups are studied. In certain cases ergodic semigroups are shown to contain small ergodic subsemigroups. Properties related to ergodicity, such as that of admitting no infinite closed invariant proper subset of the group, are discussed for semigroups of epimorphisms and of affine transformations.
\end{abstract}

1. Introduction. Dynamical systems are generally studied in two different setups. One is that of ergodic theory, where the underlying space is a probability space on which a group or semigroup of measure-preserving transformations is acting. The other is that of topological dynamics, concerned with semigroups of continuous transformations of compact spaces. The two theories merge most naturally when dealing with continuous epimorphisms, or more generally affine transformations, of compact groups.

One-parameter semigroups play a key role in both theories; such semigroups, particularly of epimorphisms, have been thoroughly investigated. Nevertheless, the study of more general actions is also needed for many problems. In this paper we deal with general semigroups of affine transformations, focusing on ergodicity and related properties.

In $\$ 2$ the definitions of ergodicity in the measure-theoretic and the topological setups are introduced. The two notions turn out to coincide for semigroups of epimorphisms. We also give a characterization of ergodicity for such a semigroup in terms of the dual action on the dual object of the group. $\$ 3$ is concerned with semigroups of affine transformations. The ergodicity of such a semigroup is shown to follow from that of the lying below semigroup of epimorphisms.

The rest of the paper deals mostly with compact abelian connected finite-dimensional groups. In $\$ 4$ it is shown that an ergodic semigroup of epimorphisms of such a group contains a finitely-generated ergodic subsemigroup. A sharper result is proved in $\$ 5$ under the additional assumption that the semigroup is commutative. We also establish a mean ergodic theorem for semigroups. $\$ 6$ studies the connection between ergodicity and the property that every finite invariant set consists of torsion elements.

Received by the editors October 18, 1983 and, in revised form, August 1, 1984.

1980 Mathematics Subject Classification. Primary 22D40, 54H15; Secondary 10F10, 28A65, 28D15, $54 \mathrm{H} 20$.

Key words and phrases. Ergodic semigroup, semigroup of epimorphisms, semigroup of affine transformations, compact group, invariant set. 
$\S 7$ is concerned with semigroups of affine transformations possessing the property that the only infinite closed invariant set is the whole group. It turns out that, under fairly mild conditions, if a semigroup of epimorphisms satisfies the property in question (or even a weaker one), then any semigroup of affine transformations lying above it satisfies this property as well.

I would like to thank E. Effros for valuable discussions on this topic.

2. Ergodic semigroups of epimorphisms of compact groups. A process is a quadruple $(\Omega, \mathscr{B}, \mu, S)$, where $(\Omega, \mathscr{B}, \mu)$ is a probability space and $S$ is a semigroup of measure-preserving transformations thereof. A flow is a pair $(\Omega, S)$, where $\Omega$ is a compact Hausdorff space and $S$ is a semigroup of continuous transformations of $\Omega$. It will be convenient to assume that the identity transformation $I$ belongs to $S$.

Definition 2.1. (1) A process $(\Omega, \mathscr{B}, \mu, S)$ is ergodic if any $S$-invariant $A \in \mathscr{B}$ satisfies $\mu(A)=0$ or $\mu(A)=1$.

(2) A flow $(\Omega, S)$ is ergodic if any closed $S$-invariant proper subset of $\Omega$ is nowhere dense.

Let us mention several properties related to ergodicity. A process $(\Omega, \mathscr{B}, \mu, S)$ is ergodic iff it admits no nonconstant $S$-invariant function. Now consider a flow $(\Omega, S)$. If the flow is ergodic then any continuous $S$-invariant function is constant, but the converse is in general false. If the set of all points whose $S$-orbit is not dense in $\Omega$ is of the first category, then the flow is ergodic. The converse is valid provided that $\Omega$ satisfies the second axiom of countability. If $S$ is a one-parameter semigroup, i.e., if it consists of the set of powers of a single transformation, then the existence of a single dense orbit implies ergodicity. For general semigroups this is not the case. In fact, one can construct a countable nonergodic semigroup of homeomorphisms of $[0,1]$ having dense orbits.

In what follows, $G$ will denote a compact Hausdorff group, $\mathscr{B}$ the $\sigma$-algebra generated by the open sets and $\mu$ the Haar measure. A transformation of the form

$$
\pi(x)=\sigma(x) \alpha, \quad x \in G,
$$

where $\sigma$ is a continuous epimorphism of $G$ and $\alpha \in G$, is called an affine transformation. An affine transformation is measure-preserving. $\pi=\sigma \alpha$ is an affine transformation lying above $\sigma$, and $\sigma$ is the epimorphism lying below $\pi$. A similar terminology will be used for a semigroup of affine transformations and the corresponding semigroup of epimorphisms. These semigroups are our object of study throughout this paper. To distinguish between the two notions of ergodicity, both of which apply for semigroups of affine transformations, we shall say that the semigroup in question, $S$, is ergodic (resp. topologically ergodic) if the process $(G, \mathscr{B}, \mu, S)$ (resp. the flow $(G, S))$ is ergodic. It will turn out that in our case these notions are equivalent.

Let us fix some notation. $\mathscr{U}$ is the set of all continuous irreducible unitary representations of $G$. $\Gamma$ is the dual object of $G$, i.e., the set of all equivalence classes of representations in $\mathscr{U}$ (see, for example, [5]). $I$ stands for the trivial element in both $\mathscr{U}$ and $\Gamma$. If $U \in \gamma \in \Gamma$, then the dimension of the representation space of $U$ is denoted by $d_{U}$, by $d_{\gamma}$ or simply by $d$ if no confusion might arise. $u_{i j}, 1 \leqslant i, j \leqslant d_{U}$, 
are the coordinate functions corresponding to $U$. An epimorphism $\sigma$ of $G$ gives rise to a dual one-to-one transformation of $\mathscr{U}$, which induces in turn a transformation on $\Gamma$. The dual of $\sigma$ will usually be denoted by $\sigma$ also. To avoid confusion we write $\sigma(x)$ for the action of $\sigma$ on $x \in G$ and $U \sigma$ or $\gamma \sigma$ for its action on representations.

THEOREM 2.1. Let $\Sigma$ be a semigroup of epimorphisms of $G$. The following conditions are equivalent:

(1) $\Sigma$ is ergodic.

(2) $\Sigma$ is topologically ergodic.

(3) $\gamma \Sigma$ is infinite for every nontrivial $\gamma \in \Gamma$.

Proof. (1) $\Rightarrow$ (2) This follows easily using the fact that the support of $\mu$ is $G$.

(2) $\Rightarrow$ (3) Suppose that for some $I \neq \gamma \in \Gamma$ the orbit $\gamma \Sigma$ is finite, say $\gamma \Sigma=$ $\left\{\gamma_{1}, \gamma_{2}, \ldots, \gamma_{s}\right\}$. Given $\delta \in \Gamma$ denote by $\chi_{\delta}$ the character corresponding to any representation $U \in \delta$. Consider the function $f: G \rightarrow \mathbf{C}$ given by $f=\sum_{i=1}^{s} \chi_{\gamma_{i}}$. It is a continuous $\Sigma$-invariant nonconstant function. The existence of such a function means that the flow $(G, \Sigma)$ is nonergodic.

(3) $\Rightarrow$ (1) Assume that $\gamma \Sigma$ is infinite for every nontrivial $\gamma \in \Gamma$ but the process $(G, \mathscr{B}, \mu, \Sigma)$ is nonergodic. Let $f$ be a nonconstant $\Sigma$-invariant function in $L^{2}(G)$. Take a nontrivial $U \in \mathscr{U}$ such that the projection of $f$ on the subspace of $L^{2}(G)$ spanned by the coordinate functions corresponding to $U$ is nonzero. Our assumptions imply the existence of a sequence $\sigma_{1}, \sigma_{2}, \ldots$ in $\Sigma$ for which the representations $U, U \sigma_{1}, U \sigma_{2}, \ldots$ belong to distinct equivalence classes. Take a maximal system $\mathscr{V}$ of nonsimilar representations in $\mathscr{U}$ containing all these representations. According to the Peter-Weyl theorem, $f$ can be expanded in $L^{2}(G)$ as follows:

$$
f=\sum_{V \in \mathscr{V}} \sum_{j, k=1}^{d_{V}} a_{V, j, k} v_{j k} .
$$

For any epimorphism $\sigma$ we have

$$
f \circ \sigma=\sum_{V \in \mathscr{V}} \sum_{j, k=1}^{d} a_{V, j, k}(v \sigma)_{j k},
$$

where $(v \sigma)_{j k}, 1 \leqslant j, k \leqslant d_{V}$, are the coordinate functions corresponding to $V \sigma$. The uniqueness of the expansion, the orthogonality relations between nonsimilar representations and the $\Sigma$-invariance of $f$ give us in particular:

$$
a_{U \sigma_{i}, j, k}=a_{U, j, k}, \quad i=1,2,3, \ldots
$$

Since $a_{U, j, k} \neq 0$ for some pair $(j, k)$ these equalities contradict the fact that $f \in L^{2}(G)$. This completes the proof.

The following lemma will be used later. First we need

DEFINITION 2.2. Let $S$ be an arbitrary semigroup and $S^{\prime}$ a subsemigroup. $S^{\prime}$ is of finite right (resp. left) index in $S$ if there exists a decomposition $S=\bigcup_{i=1}^{l} S_{i}$ and $s_{1}, \ldots, s_{l} \in S$ such that $S_{i} s_{i} \subseteq S^{\prime}$ (resp. $s_{i} S_{i} \subseteq S^{\prime}$ ) for all $1 \leqslant i \leqslant l . S^{\prime}$ is of finite index in $S$ if it is of both finite right index and finite left index in $S$. 
LEMMA 2.1. A subsemigroup of finite right index in an ergodic semigroup of epimorphisms is ergodic as well.

The proof is immediate using Theorem 2.1.

3. Ergodic semigroups of affine transformations. In this section we shall prove the following two results concerning the ergodicity of semigroups of affine transformations.

THEOREM 3.1. A semigroup of affine transformations is ergodic iff it is topologically ergodic.

THEOREM 3.2. A semigroup of affine transformations, lying above an ergodic semigroup of epimorphisms, is ergodic as well.

Proof of Theorem 3.2. Let $\Sigma$ be an ergodic semigroup of epimorphisms of $G$ and $\Pi$ a semigroup of affine transformations lying above $\Sigma$. Assume that $\Pi$ is nonergodic. Let $f$ be a nonconstant $\Pi$-invariant function in $L^{2}(G)$. Select a representation $U \in \mathscr{U}$, epimorphisms $\sigma_{1}, \sigma_{2}, \ldots \in \Sigma$ and a complete system $\mathscr{V}$ of nonsimilar representations as in the proof of Theorem 2.1. Expand $f$ in $L^{2}(G)$ :

$$
f=\sum_{V \in \mathscr{V}} \sum_{j, k=1}^{d} a_{V, j, k} v_{j k} .
$$

For any affine transformation $\pi=\sigma \alpha$ we have

$$
\begin{aligned}
f \circ \pi & =\sum_{V \in \mathscr{V}} \sum_{j, k=1}^{d} a_{V, j, k}\left(v_{j k} \circ \pi\right)=\sum_{V \in \mathscr{V} j, k=1} \sum_{V, j, k}^{d} \sum_{l=1}^{d}(v \sigma)_{j l} v_{l k}(\alpha) \\
& =\sum_{V \in \mathscr{V}} \sum_{j, l=1}^{d}\left(\sum_{k=1}^{d} a_{V, j, k} v_{l k}(\alpha)\right)(v \sigma)_{j l} .
\end{aligned}
$$

Take a sequence $\pi_{1}=\sigma_{1} \alpha_{1}, \pi_{2}=\sigma_{2} \alpha_{2}, \ldots$ in $\Pi$ lying above $\sigma_{1}, \sigma_{2}, \ldots$, respectively. Comparison of the coefficients of the coordinate functions of $V \sigma_{n}$ in the expansions of $f$ and of $f \circ \pi_{n}$ yields, due to the $\Pi$-invariance of $f$,

$$
a_{U \sigma_{n}, j, l}=\sum_{k=1}^{d} a_{U, j, k} u_{l k}\left(\alpha_{n}\right), \quad n \in \mathbf{N}, j, l=1,2, \ldots, d .
$$

Denote by $A$ (resp. $\left.A_{n}\right)$ the $d \times d$ matrix $\left(a_{U, j, l}\right)_{j, l=1}^{d}\left(\right.$ resp. $\left.\left(a_{U \sigma_{n}, j, l}\right)_{j, l=1}^{d}\right)$. The last equality can be written in terms of matrices as

$$
A_{n}=A U^{T}\left(\alpha_{n}\right), \quad n=1,2,3, \ldots
$$

(Here and elsewhere the letter $T$ stands for transpose.) Denoting $\|C\|=\max \left\{\left|c_{i j}\right|\right.$ $1 \leqslant i, j \leqslant d\}$ for a $d \times d$ matrix $C=\left(c_{i j}\right)_{i, j=1}^{d}$ we easily get

$$
\left\|A_{n}\right\| \geqslant\|A\| / d, \quad n=1,2,3, \ldots
$$

Contradicting the fact that $f \in L^{2}(G)$, the last inequality completes the proof.

Proof of TheOREM 3.1. Ergodicity obviously implies topological ergodicity so that we have to prove only the converse implication. Let $\Pi$ be a topologically 
ergodic semigroup of affine transformations. Assume $\Pi$ to be nonergodic. Denote by $\Sigma$ the lying below semigroup of epimorphisms. Take $f$ and $U$ as in the proof of Theorem 3.2. Let $\gamma \in \Gamma$ be the equivalence class of $U$. Examining the proof of Theorem 3.2 we observe that $\gamma \Sigma$ is necessarily finite.

Now we claim that for every $\delta \in \Gamma$ either $\delta \Sigma=\gamma \Sigma$ or $\delta \Sigma \cap \gamma \Sigma=\varnothing$. In fact, assuming that $\delta \Sigma \cap \gamma \Sigma \neq \varnothing$, say $\delta \sigma_{1}=\gamma \sigma_{2}$, we are going to show that $\delta \Sigma=\gamma \Sigma$. Since $\gamma \Sigma$ is finite, $\gamma \sigma_{2}^{k}=\gamma$ for some $k$. Hence $\delta \sigma_{1} \sigma_{2}^{k-1}=\gamma \sigma_{2}^{k}=\gamma$, which implies $\delta \Sigma \supseteq \gamma \Sigma$. Now put $\sigma=\sigma_{1} \sigma_{2}^{k-1}$. We have $\delta \sigma^{l+1}=\gamma \sigma^{l}=\gamma$ for some $l$, so that $\delta \sigma^{l+1}=\delta \sigma$. Thus $\delta \sigma^{\prime}=\delta$, which in turn gives $\delta=\gamma \sigma^{l-1}$. It follows that $\delta \Sigma \subseteq \gamma \Sigma$, and so finally $\delta \Sigma=\gamma \Sigma$.

Expand $f$ in $L^{2}(G)$ as a series in the coordinate functions corresponding to some complete system $\mathscr{V}$ of irreducible nonsimilar unitary representations of $G$. From the uniqueness of this expansion and the foregoing discussion we conclude that the projection of $f$ on the subspace of $L^{2}(G)$ spanned by the coordinate functions corresponding to the representations in $\gamma \Sigma$ forms a $\Pi$-invariant function also. This function is continuous and nonconstant. The existence of such a function contradicts the topological ergodicity of $\Pi$. This proves the theorem.

REMARK 3.1. From the proof of Theorem 3.1 we see that, unlike the situation for general flows, a nonergodic semigroup of affine transformations admits a continuous nonconstant invariant function. This fact also implies that if such a semigroup has a single dense orbit, then it is ergodic.

4. Finitely-generated ergodic subsemigroups. In the following two sections we shall study ergodic semigroups of epimorphisms and show that under various assumptions such semigroups contain "small" ergodic subsemigroups.

THEOREM 4.1. An ergodic semigroup of epimorphisms of a compact connected metrizable group contains a countable ergodic subsemigroup.

We defer the proof to the next section. This section will be devoted to the proof of

THEOREM 4.2. An ergodic semigroup of epimorphisms of a compact abelian connected finite-dimensional group contains a finitely-generated ergodic subsemigroup. This subsemigroup can be chosen so that its number of generators does not exceed the dimension of the group.

First we shall provide a brief description of the class of groups dealt with in the theorem and their epimorphisms. If $G$ is an $r$-dimensional compact abelian connected group, then its dual $\Gamma$ is a discrete abelian torsion-free group of rank $r$ (see, for example, [4, Chapters 23-24]). We may assume accordingly that $\mathbf{Z}^{r} \subseteq \Gamma \subseteq \mathbf{Q}^{r}$ (all endowed with discrete topology). Hence $G$ is a quotient group of $\mathbf{Q}^{r}$. Let $\sigma$ be an endomorphism of $G$. Its dual $\hat{\sigma}$ can therefore be represented by an $r \times r$ matrix with rational entries. $\hat{\sigma}$ obviously admits a unique extension to an endomorphism of $\hat{\mathbf{Q}}^{r}$, and so by duality $\sigma$ admits a unique lifting to an endomorphism of $\mathbf{Q}^{r}$. Employing the duals we see that this lifting of $\sigma$, to be denoted by $\bar{\sigma}$, is epic iff $\sigma$ is. 
Now let $\Sigma$ be a semigroup of epimorphisms of $G$ and $\bar{\Sigma}$ the semigroup consisting of their liftings to $\hat{\mathbf{Q}}^{r}$. An immediate consequence of Theorem 2.1 is

LEMMA 4.1. $\bar{\Sigma}$ is ergodic iff $\Sigma$ is.

Let us introduce several notations. Given a prime $p$ we denote by $\mathbf{Q}_{p}$ the field of $p$-adic numbers and by $\mathbf{Z}_{p}$ its subring of $p$-adic integers. The same notations apply to the additive groups of these rings. $|\cdot|_{p}$ is the $p$-adic valuation. $\mathbf{Q}_{(p)}$ is the ring of all rationals whose denominator is prime to $p . \mathbf{Z}[1 / a]$ is the ring obtained from $\mathbf{Z}$ by adjoining $1 / a$ to it, $a$ being a positive integer. $M_{n}(R)$ denotes the ring of $n \times n$ matrices with entries in the ring $R$. Given a ring $R$ with a unit element, $R^{*}$ is the multiplicative group of all invertible elements iri $R$.

LEMMA 4.2. Let $S$ be a subsemigroup of $\mathrm{GL}(r, \mathbf{Q})$ and assume that, for a certain prime $q, A \in M_{r}\left(\mathbf{Q}_{(q)}\right)$ and $|\operatorname{det} A|_{q}=1$ for every $A \in S$. Then, for any positive integer $k$, the set $S^{(k)}$ consisting of all matrices in $S$ which are congruent to I modulo $q^{k}$ forms a subsemigroup of finite index in $S$.

In fact, examine the natural homomorphism of $M_{r}\left(\mathbf{Q}_{(q)}\right)$ onto $M_{r}\left(\mathbf{Z} / q^{k} \mathbf{Z}\right)$. Our assumptions imply that the image of any $A \in S$ under this homomorphism is invertible. Taking the inverse images in $S$ of all the elements of $\operatorname{GL}\left(r, \mathbf{Z} / q^{k} \mathbf{Z}\right)$ we obtain therefore a decomposition of $S$ which satisfies the conditions of Definition 2.2 .

LEMMA 4.3. Let $S$ be a subgroup of $\mathrm{GL}(r, \mathbf{Q})$ in which every element is of finite order. Then $S$ is finite.

Proof. Assume first that for an appropriately chosen prime $q$ we have $S \subseteq$ $M_{r}\left(\mathbf{Q}_{(q)}\right)$. Take a positive integer $l$ with $q^{l}>2 r$. In view of the preceding lemma it is sufficient to show that the subgroup $S^{\prime}$, consisting of all those matrices in $S$ which are congruent to $I$ modulo $q^{\prime}$, is finite. We shall show that actually $S^{\prime}=\{I\}$. In fact, let $A \in S$. Consider the number $\operatorname{tr} A$. Being the sum of the eigenvalues of $A$, all of which are roots of unity, $\operatorname{tr} A$ is an integer. The definition of $S^{\prime}$ gives $\operatorname{tr} A=r+$ $q^{\prime} a / b$, where $q+b$. We also have $-r \leqslant \operatorname{tr} A \leqslant r$. The choice of $l$ implies therefore $\operatorname{tr} A=r$. Consequently the number 1 is the only eigenvalue of $A$. Since $A$ is of finite order it is diagonalizable. Hence $A=I$. Thus $S$ is finite.

Now to the general case. Let $\left(p_{n}\right)_{n=1}^{\infty}$ be the sequence of all primes, ordered according to increasing magnitude. Put $S^{(n)}=S \cap M_{r}\left(\mathbf{Z}\left[1 / p_{1} p_{2} \cdots p_{n}\right]\right)$. In view of the first part of the proof, $\left(S^{(n)}\right)_{n=1}^{\infty}$ is an ascending sequence of finite subgroups of $S$. Since $S=\bigcup_{n=1}^{\infty} S^{(n)}$ it only remains to show that the sequence $\left(\left|S^{(n)}\right|\right)_{n=1}^{\infty}$ is eventually constant.

To this end we first show that if $q$ is a sufficiently large prime, then $q+\left|S^{(n)}\right|$ for all $n$. If $A \in S$, then all the eigenvalues of $A$ are roots of unity of degrees not exceeding $r$ over $\mathbf{Q}$. Hence there exists an upper bound for the possible orders of these roots of unity. Every prime divisor of $\left|S^{(n)}\right|$ cannot exceed this bound.

It remains to show that if $q$ is any prime, then there exists some $k$ for which $q^{k}+\left|S^{(n)}\right|$ for every $n$. We may restrict ourselves to integers $n$ with $p_{n+1}>2 r$. Fix $n$ 
momentarily and take any prime $p>p_{n}$. Consider the natural homomorphism of $\mathrm{GL}\left(r, \mathbf{Q}_{(p)}\right)$ onto $\mathrm{GL}(r, \mathbf{Z} / p \mathbf{Z})$. Examining the first part of the proof we see that the restriction of this homomorphism to $S^{(n)}$ is one-to-one. Hence

$$
\left|S^{(n)}\right||| \operatorname{GL}(r, \mathbf{Z} / p \mathbf{Z})\left|=\left(p^{r}-1\right)\left(p^{r}-p\right) \cdots\left(p^{r}-p^{r-1}\right)\right| p^{\left(\frac{r}{2}\right)}\left(p^{r !}-1\right)^{r} .
$$

Consequently, it is sufficient to establish the existence of a positive integer $k$ and a prime $p>p_{n}$ such that $q^{k}+p^{\left(\frac{r}{2}\right)}\left(p^{r !}-1\right)^{r}$. For this we shall prove that $q^{k}+p^{r !}-1$ for infinitely many primes $p$ if $k$ is sufficiently large. Suppose first that $q$ is an odd prime. Take $k$ such that $q^{k-1}(q-1)>r$ !. Being cyclic and of order $>r$ !, the group $\left(\mathbf{Z} / q^{k} \mathbf{Z}\right)^{*}$ contains an element $x$ with $x^{r !} \neq 1$. In other words, there exists a positive integer $a$ relatively prime to $q^{k}$ such that $a^{r !} \not \equiv 1\left(\bmod q^{k}\right)$. According to Dirichlet's theorem concerning the existence of primes in arithmetic progressions [6, Theorem 6-11] there are infinitely many primes $p$ of the form $a+b q^{k}, b$ a positive integer. If $p$ is such a prime, then $q^{k}+p^{r !}-1$. The case $q=2$ is essentially the same except for the fact that, since $\left(\mathbf{Z} / 2^{k} \mathbf{Z}\right)^{*}$ is isomorphic with a direct sum of a cyclic group of order $2^{k-2}$ and a cyclic group of order 2 for $k \geqslant 3$, we have to choose $k$ so $2^{k-2}>r$ !. This proves the lemma.

Proof of Theorem 4.2. By Lemma 4.1 we may assume the underlying group to be $\hat{\mathbf{Q}}^{r}$. Our first step is proving that an ergodic group of epimorphisms of $\hat{\mathbf{Q}}^{r}$ contains a finitely-generated ergodic subsemigroup. Assume, to the contrary, that $\Sigma$ is an ergodic group of epimorphisms of $\hat{\mathbf{Q}}^{r}$ having no finitely-generated ergodic subsemigroup. Given $\gamma \in \mathbf{Q}^{r}$ and $\sigma \in \Sigma$ denote by $O_{\sigma} \gamma$ the orbit of $\gamma$ under $\sigma$. From our assumption and Theorem 2.1 we conclude that

$$
\Delta=\left\{\gamma \in \mathbf{Q}^{r}|| O_{\sigma} \gamma \mid<\infty \forall \sigma \in \Sigma\right\}
$$

is a nonzero subspace of $\mathbf{Q}^{r}$.

We claim that $\Delta$ is $\Sigma$-invariant. For this it has to be shown that if $\gamma \in \Delta$ and $\sigma, \tau \in \Sigma$, then $O_{\tau}(\gamma \sigma)$ is finite. Now $\gamma \sigma \tau^{l}=\gamma\left(\sigma \tau \sigma^{-1}\right)^{l} \sigma$ for any $l$. The definition of $\Delta$ implies $\gamma\left(\sigma \tau \sigma^{-1}\right)^{l}=\gamma$ for some $l \in \mathbf{N}$, and so $\gamma \sigma \tau^{l}=\gamma \sigma$. It follows that $\left|O_{\tau}(\gamma \sigma)\right|<$ $\infty$, whence $\Delta$ is $\Sigma$-invariant.

Given any $\sigma \in \Sigma$ we have a linear transformation $\left.\sigma\right|_{\Delta}$ of $\Delta$ defined as the restriction of $\sigma$ to $\Delta$. Let $\left.\Sigma\right|_{\Delta}=\left\{\left.\sigma\right|_{\Delta} \mid \sigma \in \Sigma\right\} .\left.\Sigma\right|_{\Delta}$ is a group of linear transformations of $\Delta$ satisfying the conditions of Lemma 4.3 and hence $\gamma \Sigma$ is finite for every $\gamma \in \Delta$. Contradicting the ergodicity of $\Sigma$, this proves that $\Sigma$ has a finitely-generated ergodic subsemigroup.

Let us show now that the same holds for any ergodic semigroup $\Sigma$. Since the underlying group is $\hat{\mathbf{Q}}^{r}, \Sigma$ consists of automorphisms. Obviously, the group generated by $\Sigma$, to be denoted by $\langle\Sigma\rangle$, is ergodic. According to the first part of the proof $\langle\Sigma\rangle$ contains a finitely-generated subsemigroup. Suppose that this subsemigroup is generated by $\tau_{1}, \tau_{2}, \ldots, \tau_{s}$, where

$$
\tau_{i}=\sigma_{i 11} \sigma_{i 12}^{-1} \sigma_{i 21} \sigma_{i 22}^{-1} \cdots \sigma_{i m 1} \sigma_{i m 2}^{-1}, \quad 1 \leqslant i \leqslant s,
$$

for some $\sigma_{i j k} \in \Sigma, 1 \leqslant i \leqslant s, 1 \leqslant j \leqslant m, 1 \leqslant k \leqslant 2$. Let $\Sigma^{\prime}$ be the subsemigroup generated by $\left\{\sigma_{i j k} \mid 1 \leqslant i \leqslant s, 1 \leqslant j \leqslant m, 1 \leqslant k \leqslant 2\right\}$. The group $\left\langle\Sigma^{\prime}\right\rangle$ generated by $\Sigma^{\prime}$ is known to be ergodic, and so $\Sigma^{\prime}$ is also ergodic. 
We may now assume that $\Sigma$ is finitely-generated. Hence a prime $q$ can be found for which $\sigma \in M_{r}\left(\mathbf{Q}_{(q)}\right)$ and $|\operatorname{det} \sigma|_{q}=1$ for every $\sigma \in \Sigma$. Let $E$ be the finite set of roots of unity whose degree over $\mathbf{Q}$ are $\leqslant r$ excluding the number 1 . Put $K=\mathbf{Q}(E)$. Let $|\cdot|_{q}$ denote some extension to $K$ to the $q$-adic valuation on $\mathbf{Q}$. Take a positive integer $s$ such that

$$
\left|(1-x)^{r}\right|_{q}>\left|q^{s}\right|_{q}, \quad x \in E .
$$

According to Lemmas 2.1 and 4.2 the subsemigroup $\Sigma^{\prime}$, consisting of all those matrices in $\Sigma$ which are congruent to $I$ modulo $q^{s}$, is also ergodic. It follows that $\Sigma$ itself may be assumed to satisfy the condition that every $\sigma \in \Sigma$ is congruent to $I$ modulo $q^{s}$. It is easy to see that the characteristic equation of any $\sigma \in \Sigma$ is of the form

$$
(1-x)^{r}+q^{s} a_{1} x^{r-1}+\cdots+q^{s} a_{r}=0,
$$

where $a_{1}, a_{2}, \ldots, a_{r} \in \mathbf{Q}_{(q)}$. By the choice of $s$ the only root of unity which can occur among the eigenvalues of $\sigma$ is 1 . We claim that for every $\gamma \in \mathbf{Q}^{r}$ there exists some $\sigma \in \Sigma$ such that $O_{\sigma} \gamma$ is infinite. In fact, given any linear transformation $\sigma$, the subspace of $\mathbf{C}^{r}$, consisting of all those points whose orbit under $\sigma$ is finite, is just the subspace spanned by all eigenvectors corresponding to eigenvalues which are roots of unity. In our case therefore if for some $\gamma \in \mathbf{Q}^{r}$ the set $O_{\sigma} \gamma$ is finite for all $\sigma \in \Sigma$, then $\gamma \sigma=\gamma$ for all $\sigma \in \Sigma$, whence $\Sigma$ is nonergodic.

To complete the proof it is sufficient to show that for every $n \leqslant r$ we can choose $\sigma_{1}, \sigma_{2}, \ldots, \sigma_{n} \in \Sigma$ such that denoting

$$
\Delta=\left\{\gamma \in \mathbf{Q}^{r} \mid \gamma \sigma_{i}=\gamma \forall 1 \leqslant i \leqslant n\right\}
$$

we have $\operatorname{dim} \Delta \leqslant r-n$. This fact is easily demonstrated by induction. Thus the proof is completed.

REMARK 4.1. Examining the proof of Lemma 4.3 one can now show that there exists an effectively computable constant $M(r)$ such that every nonergodic semigroup of epimorphisms of a compact abelian connected $r$-dimensional group admits an orbit containing at most $M(r)$ points. We note that for the infinite-dimensional torus $\mathbf{T}^{\mathbf{N}}$, for example, no such constant exists.

The last theorem is the best possible in some sense; in general an ergodic semigroup of epimorphisms of $G$ cannot be expected to contain a set of less than $\operatorname{dim} G$ epimorphisms generating an ergodic subsemigroup. This is seen in

EXAMPLE 4.1. Let $\Sigma$ be the semigroup of all epimorphisms of $\mathbf{T}^{r}$ of the form $\sigma_{a_{1} a_{2} \cdots a_{r}}, a_{1}, a_{2}, \ldots, a_{r} \in \mathbf{Z}$, where

$$
\sigma_{a_{1} a_{2} \ldots a_{r}}\left(x_{1}, x_{2}, \ldots, x_{r}\right)^{T}=\left(x_{1}+a_{1} x_{r}, x_{2}+a_{2} x_{r}, \ldots, a_{r} x_{r}\right)^{T}(\bmod .1),
$$

$\left(x_{1}, x_{2}, \ldots, x_{r}\right)^{T} \in \mathbf{T}^{r}$. It is easy to verify that $\Sigma$ is ergodic but no $(r-1)$-generated subsemigroup is such.

5. One-parameter ergodic subsemigroups. In this section we shall prove a sharpened version of Theorem 4.2 for commutative semigroups. In the course of the proof we shall obtain Theorem 4.1 as a by-product. 
THEOREM 5.1. A commutative semigroup of epimorphisms of a compact abelian connected finite-dimensional group is ergodic iff it contains an ergodic epimorphism.

LEMMA 5.1. Let $\Sigma$ be an ergodic semigroup of epimorphisms of a compact connected group $G$. Then for every nontrivial $\gamma_{i j} \in \Gamma, 1 \leqslant i \leqslant k, 1 \leqslant j \leqslant 2$, there exists some $\sigma \in \Sigma$ with $\gamma_{i 1} \sigma \neq \gamma_{i 2}, 1 \leqslant i \leqslant k$.

Proof. Assume, to the contrary, that there exist nontrivial $\gamma_{i j} \in \Gamma, 1 \leqslant i \leqslant k$, $1 \leqslant j \leqslant 2$, such that for every $\sigma \in \Sigma$ we have $\gamma_{i 1} \sigma=\gamma_{i 2}$ for some $1 \leqslant i \leqslant k$. For any $\varepsilon>0$ define two closed sets in $G$ as follows:

$$
\begin{aligned}
& A_{\varepsilon}=\bigcap_{i=1}^{k}\left\{x \in G|| \chi_{\gamma_{i 1}}(x)-d \mid \leqslant \varepsilon\right\}, \\
& B_{\varepsilon}=\bigcup_{i=1}^{k}\left\{x \in G|| \chi_{\gamma_{i 2}}(x)-d \mid \leqslant \varepsilon\right\} .
\end{aligned}
$$

Our assumptions obviously imply $\Sigma A_{\varepsilon} \subseteq B_{\varepsilon}$. To arrive at a contradiction to the ergodicity of $\Sigma$ it is sufficient therefore to show that $B_{\varepsilon}$ is a proper subset of $G$ for a sufficiently small $\varepsilon>0$. To this end it suffices to prove that if $\gamma \in \Gamma$ is nontrivial, then

$$
\mu\left(\left\{x \in G|| \chi_{\gamma}(x)-d_{\gamma} \mid \leqslant \varepsilon\right\} \rightarrow 0 \text { as } \varepsilon \rightarrow 0 .\right.
$$

In fact, assume this to be false. If $U \in \gamma$, then

$$
\begin{gathered}
\mu(\{x \in G \mid U(x)=I\})=\mu\left(\left\{x \in G \mid \chi_{\gamma}(x)=d_{\gamma}\right\}\right) \\
=\lim _{\varepsilon \rightarrow 0} \mu\left(\left\{x \in G|| \chi_{\gamma}(x)-d_{\gamma} \mid \leqslant \varepsilon\right\}\right)>0 .
\end{gathered}
$$

Given any matrix $A$ in the image of $U$, the set $\{x \in G \mid U(x)=A\}$ is a coset of $\{x \in G \mid U(x)=I\}$, whence both sets have the same measure. Consequently, the image of $U$ is finite. Since $G$ is connected this actually means that $U$ is constant, contradicting the nontriviality of $\gamma$. This proves the lemma.

We now need the notion of an ergodic sequence of transformations. Let $\left(T_{n}\right)_{n=1}^{\infty}$ be a sequence of measure-preserving transformations of a probability space $(X, \mathscr{B}, \mu)$. The sequence is ergodic if

$$
\frac{1}{N} \sum_{n=1}^{N} T_{n} f \underset{N \rightarrow \infty}{\stackrel{L^{2}}{\rightarrow}} \int_{X} f d \mu \quad \text { for every } f \in L^{2}(X, \mathscr{B}, \mu) .
$$

This definition is equivalent with the one given in [2] in view of the basic results there.

Proposition 5.1. A semigroup of epimorphisms of a compact connected metrizable group is ergodic iff it contains an ergodic sequence.

Proof. The "if" part is immediate. For the converse direction, let $\Sigma$ be an ergodic semigroup of epimorphisms of a compact connected metrizable group $G$. Since $G$ is metrizable, $L^{2}(G)$ is separable so that $\Gamma$ is countable, say $\Gamma=\left\{I, \gamma_{1}, \gamma_{2}, \ldots\right\}$. Construct a sequence $\left(\sigma_{n}\right)_{n=1}^{\infty}$ inductively as follows. Select $\sigma_{1} \in \Sigma$ arbitrarily. 
Suppose $\sigma_{1}, \sigma_{2}, \ldots, \sigma_{n}$ have already been defined. According to Lemma 5.1 there exists some $\sigma \in \Sigma$ such that $\gamma_{i} \sigma \neq \gamma_{i} \sigma_{j}$ for all $1 \leqslant i \leqslant n+1,1 \leqslant j \leqslant n$. Take $\sigma_{n+1}=\sigma$. Now consider the sequence $\left(\gamma_{l} \sigma_{n}\right)_{n=1}^{\infty}$ for any $l$. Discarding its $l$ initial terms we obtain a sequence which assumes each value just once. The results of [2] imply that $\left(\sigma_{n}\right)_{n=1}^{\infty}$ is ergodic (in fact, even strongly mixing). This proves the proposition.

Corollary. A countable semigroup of epimorphisms of a compact connected metrizable group is ergodic iff it can be ordered so as to form an ergodic sequence.

In fact, if $\Sigma$ is ergodic we can form an ergodic sequence out of some of its elements. Now we insert all the other elements into sufficiently distant places in this sequence. The new sequence gives an ergodic ordering of $\Sigma$.

Proof of Theorem 4.1. Take an ergodic sequence of epimorphisms in the given semigroup. The subsemigroup generated by all the epimorphisms in this sequence is a countable ergodic semigroup.

Proof of Theorem 5.1. We have to prove only that if $\Sigma$ is ergodic, then it contains an ergodic epimorphism. Arrange the elements of $\Sigma$ as an ergodic sequence $\left(\sigma_{n}\right)_{n=1}^{\infty}$. Suppose no element of $\Sigma$ is ergodic. Then every $\sigma_{n}$ has some root of unity among its eigenvalues. It follows that some fixed root of unity $\lambda$ is an eigenvalue of $\sigma_{n}$ for a set of positive upper density of numbers $n$. The results of [2] imply then that $\left(\sigma_{n}\right)_{n=1}^{\infty}$ is nonergodic. The contradiction proves the theorem.

The following example demonstrates that our results in the last two sections fail to be true if some of the underlying assumptions are dropped.

EXAMPLE 5.1. Given a sequene $\vec{a}=\left(a_{n}\right)_{n=1}^{\infty}$ of nonzero integers we define an epimorphism $\sigma_{\vec{a}}$ of $\mathbf{T}^{\mathbf{N}}$ by

$$
\sigma_{\dot{a}}\left(x_{1}, x_{2}, \ldots\right)^{T}=\left(a_{1} x_{1}, a_{2} x_{2}, \ldots\right)^{T}(\bmod .1), \quad\left(x_{1}, x_{2}, \ldots\right)^{T} \in \mathbf{T}^{\mathbf{N}} \text {. }
$$

Let $F$ be the set of all sequences $\vec{a}$ with $a_{n}=1$ for sufficiently large $n$. Take $\Sigma=\left\{\sigma_{\vec{a}} \mid\right.$ $\vec{a} \in F\} . \Sigma$ is a commutative ergodic semigroup but no finitely-generated subsemigroup is ergodic. Thus, the condition concerning the finite-dimensionality of the group is essential in both Theorems 4.2 and 5.1. If instead of $\mathbf{T}^{\mathbf{N}}$ we consider $\mathbf{T}^{I}$, where $I$ is uncountable, and define $\Sigma$ analogously, then we obtain an ergodic semigroup containing no countable ergodic subsemigroup, so that the metrizability condition in Theorem 4.1 cannot be dropped.

EXAMPLE 5.2. The semigroup consisting of all rational rotations of $\mathbf{T}$ is ergodic but no finitely-generated subsemigroup is. Thus the semigroups of epimorphisms in Theorems 4.2 and 5.1 cannot be replaced by semigroups of affine transformations.

6. Finite invariant sets. In this section we shall be interested in the conditions under which the property now to be defined is satisfied.

Definition 6.1. A semigroup of epimorphisms $\Sigma$ of a compact abelian group $G$ is an $F T$ semigroup if every finite $\Sigma$-invariant set consists of torsion elements of $G$. (FT - Finite is Torsion.) 
The importance of studying the FT property stems from its connection with the ID property, discussed in the next section. The main result of this section is

THEOREM 6.1. A commutative semigroup of epimorphisms of a compact abelian connected finite-dimensional group is an FT semigroup iff it is ergodic.

Before the proof can be presented some more information concerning groups of the type considered in the theorem is needed. (For more details see [1, §§IV.1, V.1] and the references mentioned there.) Let $G$ be such a group and $\Gamma=\hat{G}$. As we have seen, it may be assumed that $\mathbf{Z}^{r} \subseteq \Gamma \subseteq \mathbf{Q}^{r}$. These embeddings give rise to dual epimorphisms $\hat{\mathbf{Q}}^{r} \rightarrow G \rightarrow \mathbf{T}^{r}$. Let us describe $\hat{\mathbf{Q}}$. Put $\boldsymbol{P}=\left\{p_{0}, p_{1}, p_{2}, \ldots\right\}$, where $p_{0}=\infty$ and $p_{1}, p_{2}, \ldots$ are all the primes. Denote by $\prod_{j=1}^{\prime \infty} \mathbf{Q}_{p_{j}}$ the local direct product of the groups $\mathbf{Q}_{p_{j}}$ with respect to the compact open subgroups $\mathbf{Z}_{p_{j}}$. Agreeing that $Q_{\infty}=\mathbf{R}$ and $\mathbf{Z}_{\infty}=[0,1]$ we set $\Pi_{p \in P}^{\prime} \mathbf{Q}_{p}=\mathbf{R} \times \prod_{j=1}^{\prime \infty} \mathbf{Q}_{p_{j}}$. Consider the embedding $i: \mathbf{Q} \rightarrow \prod_{p \in P}^{\prime} \mathbf{Q}_{p}$ given by $i(s)=(s, s, \ldots), s \in \mathbf{Q}$. The group $\Pi_{p \in P}^{\prime} \mathbf{Q}_{p}$ is self-dual and hence the dual homomorphism

$$
\pi=\hat{i}: \prod_{p \in P}{ }^{\prime} \mathbf{Q}_{p} \rightarrow \hat{\mathbf{Q}}
$$

exhibits $\hat{\mathbf{Q}}$ as a quotient group of $\Pi_{p \in P}^{\prime} \mathbf{Q}_{p}$. An explicit representation of $\hat{\mathbf{Q}}$ is given by the set $\prod_{p \in P} \mathbf{Z}_{p}$ (certain pairs of points being identified). If the sequences $\left(x_{p}\right)_{p \in P},\left(y_{p}\right)_{p \in P} \in \prod_{p \in P} \mathbf{Z}_{p}$ correspond to the points $x, y \in \hat{\mathbf{Q}}$, then the sequence $\left(z_{p}\right)_{p \in P}$ corresponding to $z=x+y$ is given by

$$
z_{p}= \begin{cases}x_{\infty}+y_{\infty}-\left[x_{\infty}+y_{\infty}\right], & p=\infty, \\ x_{p}+y_{p}+\left[x_{\infty}+y_{\infty}\right], & p \neq \infty .\end{cases}
$$

Now we can prove the theorem.

Proof. Suppose $\Sigma$ is ergodic. By Theorem 5.1 there exists an ergodic $\sigma \in \Sigma$. The proof of Lemma II.15 in [1] carries over to our case to show that a finite set, invariant under an ergodic epimorphism, necessarily consists of torsion elements. Hence $\Sigma$ is an FT semigroup.

For the converse direction assume $\Sigma$ to be nonergodic. Employing Theorem 5.1 we infer that the semigroup $\Sigma^{T}$, consisting of the transposes of all matrices in $\Sigma$, is nonergodic as well. Consequently we can choose a nonzero row vector $\gamma \in \mathbf{Z}^{r}$ such that $\gamma \in \Sigma^{T}$ is finite. Put $v_{\alpha}=\left(\alpha \gamma^{T}, 0,0, \ldots\right) \in \prod_{p \in P}^{\prime} \mathbf{Q}_{p}^{r}$ for $\alpha \in \mathbf{R}$. $\Sigma v_{\alpha}$ is obviously finite for any $\alpha \in \mathbf{R}$. Now if $\alpha$ is irrational, then $v_{\alpha}$ projects into a nontorsion element in $\mathbf{T}^{r}$. Hence the projection of $v_{\alpha}$ in $G$ is also a nontorsion element. This completes the proof.

EXAMPLE 6.1. The semigroup $\Sigma$ of epimorphisms discussed in Example 4.1 is ergodic but not FT. The semigroup $\Sigma^{T}$, on the other hand, is FT but not ergodic. Thus for noncommutative semigroups there is no necessary connection between ergodicity and the FT property.

EXAMPLE 6.2. The shift transformation on $\mathbf{T}^{\mathbf{Z}}$, although ergodic, admits many nontorsion periodic points. 
7. ID semigroups of affine transformations. In this section we study the following property.

DEFINITION 7.1. A semigroup $\Pi$ of affine transformations of a compact abelian metrizable group is an $I D$ semigroup if the only infinite closed $\Pi$-invariant subset of $G$ is $G$ itself.

Apart from the dynamical aspect, the concept of an ID semigroup is of interest from a number-theoretical viewpoint. For example, a semigroup $\Sigma$ of endomorphisms of $\mathbf{T}$ is ID iff $\{\sigma \in \Sigma \mid \sigma>0\}$ is not contained in the set of powers of a single integer [3, Chapter IV]. Thus, given such a multiplicative semigroup of integers $\Sigma$, the set $\Sigma \alpha$ is dense modulo 1 for every irrational $\alpha$. In [1] a full characterization was given of commutative ID semigroups of epimorphisms of compact abelian connected finite-dimensional groups. Here the ID property will be examined for semigroups of affine transformations. It will be shown that, under various assumptions, a semigroup of affine transformations lying above an ID semigroup of epimorphisms is ID itself. Thus, applying the following theorem to semigroups of affine transformations of $\mathbf{T}$, acting on correspondingly chosen points, one obtains sets of integers $\Delta$ (not semigroups) having the property that $\Delta \alpha$ is dense modulo 1 for any irrational $\alpha$.

THEOREM 7.1. A semigroup of affine transformations of a compact abelian connected finite-dimensional group, lying above a commutative ID semigroup of epimorphisms, is ID as well.

For the time being $G$ will denote a compact abelian metrizable group. $\Sigma$ is always a semigroup of epimorphisms of $G$ and $\Pi$ a semigroup of affine transformations lying above $\Sigma$. In the course of our discussion we shall make use of several ideas introduced by Furstenberg [3] while studying the ID property for semigroups of epimorphisms of $\mathbf{T}$.

Definition 7.2. (1) A closed $\Sigma$-invariant set $A$ in $G$ is $\Sigma$-restricted if $A+B=G$, $B$ a closed $\Sigma$-invariant set, implies $B=G$.

(2) $\Sigma$ is an $M R$ semigroup if every $\Sigma$-minimal set is $\Sigma$-restricted.

DEFINITION 7.3. $\Sigma$ is an $I D_{0}$ semigroup if the only closed $\Sigma$-invariant set which contains 0 as a nonisolated point is $G$.

LEMMA 7.1. If $\Sigma$ is ID $D_{0}$, then it is $F T$.

Proof. Let $F$ be a finite $\Sigma$-invariant set. For every positive integer $n$ the set $n F$ is also $\sum$-invariant. Given any neighborhood $U$ of 0 there exists some $n \in \mathbf{N}$ such that $n F \subseteq U$. Hence we can take a sequence $\left(n_{k}\right)_{k=1}^{\infty}$ with $n_{k} F \rightarrow\{0\}$ as $k \rightarrow \infty$ (in the Hausdorff metric). The set $A=\overline{\bigcup_{k=1}^{\infty} n_{k} F}$ is a closed $\Sigma$-invariant proper subset of $G$ and, provided $F$ contains a nontorsion element, contains 0 as a nonisolated point. Hence $F$ consists of torsion elements, which proves the lemma.

Lemma 7.2. If $\Sigma$ is ID $D_{0}$, then it is ergodic.

The lemma follows from the fact that nonergodic semigroups of epimorphisms admit continuous nonconstant invariant functions. 
Proposition 7.1. If $\Sigma$ is ID and there exists a finite $\Pi$-invariant set, then $\Pi$ is ID.

Proof. Let $A$ be an infinite closed $\Pi$-invariant set and $B$ a finite $\Pi$-invariant set. The set $A-B=\{a-b \mid a \in A, b \in B\}$ is $\Sigma$-invariant. Since $\Sigma$ is ID we get $A-B=G$. Thus a finite union of translates of $A$ is the whole group, so that $A$ has a nonempty interior. By Lemma 7.2 and Theorem 3.2 $\Pi$ is ergodic, whence $A=G$. This proves the proposition.

Definition 7.4. A flow $(X, S)$ is an $\mathscr{F}$ flow if there exists a descending sequence $\left(S^{(k)}\right)_{k=1}^{\infty}$ of subsemigroups of $S$, with $I \in S^{(k)}$ for each $k$, such that:

(1) $\left(X, S^{(k)}\right)$ is ergodic for every $k$.

(2) $S / S^{(k)}$ has a finite group structure in the following sense: $S$ decomposes into a disjoint union $S=\bigcup_{i=1}^{n_{k}} S_{i}^{(k)}\left(S_{1}^{(k)}=S^{(k)}\right)$ such that for every $1 \leqslant i, j \leqslant n_{k}$ there exists some $1 \leqslant h \leqslant n_{k}$ with $S_{i}^{(k)} S_{j}^{(k)} \subseteq S_{h}^{(k)}$. The collection $\left\{S_{i}^{(k)} \mid 1 \leqslant i \leqslant n_{k}\right\}$ forms a finite group under the operation $\circ$ defined by $S_{i}^{(k)} \circ S_{j}^{(k)}=S_{h}^{(k)}$.

(3) $\bigcup_{k=1}^{\infty}\left\{x \in X \mid S^{(k)} x=\{x\}\right\}=X$.

Note that $S^{(k)}$ is in particular of finite index in $S$ in the sense of Definition 2.2.

THEOREM 7.2 [1, Theorem I.2]. If $(G, \Sigma)$ is an $\mathscr{F}$ flow, then $\Sigma$ is $M R$.

For the rest of this section $G$ will denote a compact abelian connected finitedimensional group with dual $\Gamma, \mathbf{Z}^{r} \subseteq \Gamma \subseteq \mathbf{Q}^{r}$.

LEMMA 7.3. $\Sigma$ is ID iff it is both $I D_{0}$ and $M R$.

The "only if" part is straightforward. The "if" part goes, employing our previous results, exactly as the proof of Proposition II.7 and the subsequent conclusion of Theorem II.1 in [1].

LEMMA 7.4. If $\Sigma$ is $I D_{0}$ and $\Sigma^{\prime}$ is of finite index in $\Sigma$, then $\Sigma^{\prime}$ is $I D_{0}$ as well.

Using Lemmas 2.1 and 7.2 the proof is immediate.

THEOREM 7.3. If $\Sigma$ is an $I D_{0} \mathscr{F}$-flow, then $\Pi$ is ID.

Proof. That $\Sigma$ itself is ID follows from Theorem 7.2 and Lemma 7.3. Now take a sequence $\left(\Sigma^{(k)}\right)_{k=1}^{\infty}$ of subsemigroups of $\Sigma$ such that the conditions of Definition 7.4 are satisfied. Let $\Pi^{(k)}$ be the subsemigroup of $\Pi$ consisting of those transformations lying above any $\sigma \in \Sigma^{(k)}$. Put $F_{k}=\left\{x \in G \mid \Sigma^{(k)} x=\{x\}\right\}$. Select a dense sequence $\left(x_{k}\right)_{k=1}^{\infty}$ in $G$. We may assume that $x_{k} \in F_{k}$ for all $k$. Let $E$ be an infinite closed $\Pi$-invariant set. $E-E$ is an infinite closed $\Sigma$-invariant set, so that $E-E=G$. Hence the set $E_{1}=\left\{x \in E \mid x+x_{1} \in E\right\}$, which is readily seen to be a closed $\Pi^{(1)}$-invariant set, is nonempty. In general, for each $n$ the set $E_{n}=\{x \in E \mid$ $\left.x+x_{i} \in E \forall 1 \leqslant i \leqslant n\right\}$ is a closed $\Pi^{(n)}$-invariant set. Assume that $E_{n} \neq \varnothing$ for some $n$. If $E_{n}$ is finite, then by Proposition 7.1 and Lemma 7.4 $\Pi^{(n)}$ is ID, so that we are done. If $E_{n}$ is infinite, then $E_{n}-E_{n}$ is an infinite closed $\Sigma^{(n)}$-invariant set whence $E_{n}-E_{n}=G$. It follows therefore that the set $E_{n+1}=\left\{x \in E \mid x+x_{i} \in E\right.$ $\forall 1 \leqslant i \leqslant n+1\}$ is nonempty. As we see, either the process terminates at some stage, in which case $\Pi$ is ID, or the process continues indefinitely. In the latter case we get a point $x \in E$ with $x+x_{n} \in E$ for all $n$. Hence in this case $E$ is dense, which implies $E=G$. This completes the proof. 
The theorem points at the importance of the concept of $\mathscr{F}$-flows for the study of the ID property. We intend therefore to look for conditions on semigroups of epimorphisms which are sufficient to guarantee that these form $\mathscr{F}$-flows. In the following lemmas $q$ denotes an arbitrary fixed prime. For a positive integer $n$ we put $G[n]=\{x \in G \mid n x=0\}$. First we need

LEMMA 7.5. If $\Gamma \subseteq \mathbf{Q}_{(q)}^{r}$, then $\overline{\bigcup_{k=1}^{\infty} G\left[q^{k}\right]}=G$.

The lemma is proved just as Lemma II.1b in [1].

Proposition 7.2. If $\Gamma \subseteq \mathbf{Q}_{(q)}^{r}$, $\Sigma$ is ergodic and $|\operatorname{det} \sigma|_{q}=1$ for every $\sigma \in \Sigma$, then $(G, \Sigma)$ is an $\mathscr{F}$ flow.

The proof follows that of Proposition II.6 of [1], so that only a brief outline will be provided. For every $k$ we define $\Sigma^{(k)}$ as the subsemigroup of $\Sigma$ consisting of those matrices which are congruent to $I$ modulo $q^{k}$. Then $\Sigma^{(k)}$ is ergodic and we easily verify that the second condition in the definition of $\mathscr{F}$-flows is also satisfied. To establish the third condition it is sufficient, according to the preceding lemma, to show that if $\sigma \in \Sigma^{(k)}$ and $x \in G\left[q^{k}\right]$, then $\sigma x=x$. Write $\sigma=I+q^{k} \tau$ with $\tau \in$ $M_{r}\left(\mathbf{Q}_{(q)}\right)$. We have to prove that $\tau$ represents an endomorphism of $G$. For an appropriately chosen positive integer $a$, which may be assumed to be relatively prime to $q, a \tau$ is an integer matrix and hence represents an endomorphism of $G$. Take integers $l$ and $m$ with $l a+m q^{k}=1$. Then $\tau=l(a \tau)+m\left(q^{k} \tau\right)$ is an endomorphism of $G$. This completes the proof.

Theorem 7.3 and Proposition 7.2 taken together provide an ample supply of semigroups of epimorphisms for which the $\mathrm{ID}_{0}$ property implies the ID property to be valid for any lying above semigroup of affine transformations. We point out two special cases.

CorollaRY I. If $\Gamma \subseteq \mathbf{Q}_{(q)}^{r}$ and $\Sigma$ is an ID group, then $\Pi$ is $I D$.

COROllARY II. If $\Gamma \subseteq \mathbf{Z}[1 / a]^{r}$ for some positive integer $a$ and $\Sigma$ is a finitely-generated $I D_{0}$ semigroup, then $\Pi$ is $I D$.

Proof of Theorem 7.1. Most of the proof relies on considerations described in detail in [1] and so we shall only sketch it.

Being a commutative ID semigroup, $\Sigma$ is ergodic and hence contains an ergodic $\sigma$. We first claim that $\sigma$ may be assumed to lie in $\Pi$. For this we notice that, in general, if a set $E$ is invariant under an affine transformation $\rho$ lying above an epimorphism $\tau$, say $\rho(x)=\tau(x)+\alpha$, then the set $E+\beta$ is $\rho^{\prime}$-invariant, where $\rho^{\prime}(x)=\tau(x)+(\alpha$ $+\beta-\tau(\beta))$. Now if 1 is not an eigenvalue of $\tau$, which is in particular the case if $\tau$ is ergodic, then $I-\tau$ is epic, whence for an appropriately chosen $\beta$ the set $E+\beta$ is $\tau$-invariant. Returning to our situation we assume accordingly that $\sigma \in \Pi$.

As in the proofs of Theorems IV.1 and V.1 in [1] we can represent $G$ as an inverse limit of groups $G_{n}$, each satisfying the conditions of Corollary II, and find corresponding finitely-generated subsemigroups $\Sigma^{(n)}$ of $\Sigma$ inducing ID semigroups of epimorphisms on these groups. In view of Corollary II the corresponding subsemigroups $\Pi^{(n)}$ of $\Pi$ also induce ID semigroups on the groups $G_{n}$. Suppose $E$ is an 
infinite closed $\Pi$-invariant set in $G$. Let $E_{n}$ be the image of $E$ under the projection of $G$ onto $G_{n}$. Suppose $E_{n}$ is finite for all $n$. Since $E_{n}$ is invariant under the ergodic epimorphism induced by $\sigma$ it consists of torsion elements of $G_{n}$. Employing Lemma V.2 of [1] we infer that there exists a positive integer $l$ such that $E_{n} \subseteq G_{n}[l]$ for all $n$. This implies $E \subseteq G[l]$ which, by [1, Lemma V.1], contradicts the infinity of $E$. It follows that $E_{n}$ is infinite for some $n=n_{0}$ and so for every $n \geqslant n_{0}$. Invoking Corollary II we get $E_{n}=G_{n}$ for all $n$, and thus $E=G$. This proves the theorem.

\section{REFERENCES}

1. D. Berend, Multi-invariant sets on compact abelian groups, Trans. Amer. Math. Soc. 286 (1984), 505-535.

2. D. Berend and V. Bergelson, Ergodic and mixing sequences of transformations, Ergodic Theory and Dynamical Systems (to appear).

3. H. Furstenberg, Disjointness in ergodic theory, minimal sets, and a problem in diophantine approximation, Math. Systems Theory 1 (1967), 1-49.

4. E. Hewitt and K. A. Ross, Abstract harmonic analysis. Vol. I, Springer-Verlag, Berlin, 1963.

5. __ Abstract harmonic analysis. Vol. II, Springer-Verlag, Berlin, 1970.

6. W. J. Le Veque, Topics in number theory. Vol. II, Addison-Wesley, Reading, Mass., 1956.

Department of Mathematics, University of California, Los Angeles, California 90024

Current address: Mathematical Sciences Research Institute, Berkeley, California 94720 